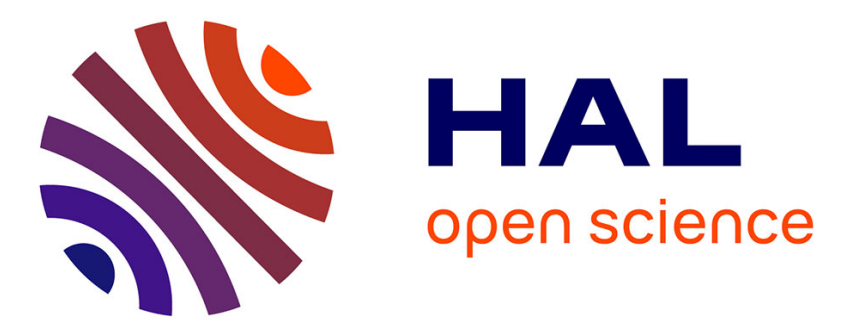

\title{
In search of principles for a Theory of Organisms
}

Giuseppe Longo, Maël Montévil, Carlos Sonnenschein, Ana Soto

\section{To cite this version:}

Giuseppe Longo, Maël Montévil, Carlos Sonnenschein, Ana Soto. In search of principles for a Theory of Organisms. Journal of Biosciences, 2015, 40 (5), 10.1007/s12038-015-9574-9 . hal-01253484

\section{HAL Id: hal-01253484 \\ https://hal.science/hal-01253484}

Submitted on 11 Jan 2016

HAL is a multi-disciplinary open access archive for the deposit and dissemination of scientific research documents, whether they are published or not. The documents may come from teaching and research institutions in France or abroad, or from public or private research centers.
L'archive ouverte pluridisciplinaire HAL, est destinée au dépôt et à la diffusion de documents scientifiques de niveau recherche, publiés ou non, émanant des établissements d'enseignement et de recherche français ou étrangers, des laboratoires publics ou privés.

\section{(이) $\$$}

Distributed under a Creative Commons Attribution - NonCommercial - NoDerivatives| 4.0 


\title{
Review
}

\section{In search of principles for a Theory of Organisms}

\author{
Giuserpe Longo $^{1,2}$, Mä̈l Montévil ${ }^{2,3}$, CARlos Sonnenschein ${ }^{2,4}$ and Ana M Soto ${ }^{1,2, *}$ \\ ${ }^{1}$ Centre Cavaillès, République des Savoirs, CNRS USR3608, Collège de France et Ecole \\ Normale Supérieure, Paris, France \\ ${ }^{2}$ Department of Integrative Physiology and Pathobiology, Tufts University School of Medicine, \\ Boston, MA, USA \\ ${ }^{3}$ CNRS, UMR8590 IHPST, Paris, France \\ ${ }^{4}$ Centre Cavaillès, École Normale Supérieure, and Institut d'Etudes Avancees de Nantes, Nantes, France \\ *Corresponding author (Fax, 617-636-3971; Email, ana.soto@tufts.edu)
}

\begin{abstract}
Lacking an operational theory to explain the organization and behaviour of matter in unicellular and multicellular organisms hinders progress in biology. Such a theory should address life cycles from ontogenesis to death. This theory would complement the theory of evolution that addresses phylogenesis, and would posit theoretical extensions to accepted physical principles and default states in order to grasp the living state of matter and define proper biological observables. Thus, we favour adopting the default state implicit in Darwin's theory, namely, cell proliferation with variation plus motility, and a framing principle, namely, life phenomena manifest themselves as non-identical iterations of morphogenetic processes. From this perspective, organisms become a consequence of the inherent variability generated by proliferation, motility and self-organization. Morphogenesis would then be the result of the default state plus physical constraints, like gravity, and those present in living organisms, like muscular tension.
\end{abstract}

[Longo G, Montévil M, Sonnenschein C and Soto AM 2015 In search of principles for a Theory of Organisms. J. Biosci.] DOI 10.1007/s12038015-9574-9

Whether you can or cannot observe a thing depends on the theory which you use. It is the theory which decides what can be observed.

- Einstein, oral remark quoted in Salam (1990)

\section{Motivation}

Biologists acknowledge a crisis in their midst: On the one hand, reams of data acquired from a reductionist perspective (for example, 'transcriptomics') do not provide the anticipated understanding of the subject matter of their interest; on the other hand, the application of mathematical modeling has not helped much either. This is due in part to the preponderance of 'pragmatic systems biology,' a practice that emphasizes large-scale molecular interactions which is technology-driven and does not claim explicit theory commitments (O’Malley and Dupre 2005).

Fifty years ago the notions of program, information and signal borrowed from mathematical theories seemed to resolve the differences between physics and biology made explicit by Kant: teleology, now under the guise of a program, became acceptable to reductionists who claimed that biology could be reduced to physics and chemistry (Jacob 1974). However, the notions of program, information and signal borrowed from the mathematical theories of information have been hindering progress in biology (Longo et al. 2012a). These notions require searching for biological specificity at the molecular level which is not coherent with that of biomechanics, another

Keywords. Autopoiesis; biological organization; criticality; default state; development; emergence; mathematical symmetries 
important contributor to phenotypes. Yet biomechanics became prominent in several biological fields including cancer research. Indeed, tumours are palpable, a fact obviously related to their being more rigid than the surrounding normal tissue. Thus, technical knowhow is being introduced together with new operational definitions which may not be coherent with the ones already in use. ${ }^{1}$

The above-referred practice contributes to the feeling that biological complexity is unfathomable and that generalizations and global concepts are unhelpful. ${ }^{2}$ Thus, the theoretical bases underlying the experimental programs being pursued remain only implicit. Meanwhile, the explosion of data continues unabated having neither sound theoretical bases nor an adequate language to make sense of them. While acknowledging the immense complexity of organisms, we dare to think that what Darwin achieved for evolution could eventually materialize for ontogenesis: this will be achieved through the elaboration of an appropriate theoretical framework.

\section{The role of theories}

At the beginning of the Scientific Revolution, scientists thought that they had direct access to their outside world: God's will was to make nature intelligible to creatures like us. The separation of science and religion was a long and complex process: Kant's philosophy and Darwin's theory were major contributors to this separation. Ever since, scientists acknowledge that they are inside the world they wish to observe and study. As a result of this realization, objectivity had to be constructed through scientific theories that would provide intelligibility principles to frame observations, experiments and explanations. In the 16th and 17th centuries, physicists developed theories that provided an accurate description of phenomena of the inert. In this theory-rich context, scientists were aware that theories determine which are the proper observables while, conversely, the choice of observables was a major theoretical commitment. For example, the decision to investigate the relationship between pressure, volume and temperature well before considering the atomic structure of gases (17th century,

\footnotetext{
${ }^{1}$ In operationalism, scientific terms are defined by the experimental operations which determine their applicability. For example, epidermal growth factor is a misnomer because it does not induce proliferation. However, it induces cell spreading in one assay: when cells spread, the circumference of the colony is longer, and as only the cells in the periphery proliferate, EGF in this assay seems to increase cell number. When given in vivo, it induces early eye opening, a process characterized by cell death. So, in one operational definition it increases cell number, in the other, it increases cell death.

${ }^{2}$ For example, some biologists believe that there are exceptions to every rule, and that there are no valid 'principles', 'rules' or overarching concepts.
}

Boyle and Mariotte), allowed for the development of a theory at that level of observability. Further work at the macroscopic level originated modern thermodynamics (TD), another major achievement of the 19th century; this was well before Boltzmann's unification with an atomistic perspective. Notwithstanding, atoms and molecules are proper observables in another physical theory, quantum mechanics (QM).

When living entities die, they decompose into particles of inert matter, and in turn, living organisms assemble the same inert matter in novel ways. The emergence of these novelties requires suitable theoretical constructs (Longo and Montévil 2012; Longo and Montévil 2014; Saçlioglu et al. 2014). That is, besides the already acknowledged physical principles and default states, additional principles and theoretical requirements are needed to describe proper biological observables, such as phenotypes. These extensions of physical laws into biology must be compatible with physical theory about inert matter, i.e. organisms should not violate the laws of thermodynamics, gravity or the quantum properties of their component particles. Yet, these principles may not suffice to make the biological dynamics intelligible at the phenotypic level.

In biology, other than Darwin's theory of evolution (1859), the creation of global theories has not been as successful as in physics (Soto and Sonnenschein 2012). Among other subjects, Darwin's theory on the origin of species addressed common descent, encompassed a long timeframe and provided an adequate explanation of phylogeny. The fundamental principles in Darwin's theory are (a) descent with modification and (b) natural selection. However, biology has yet to produce a theory of organisms that would encompass ontogeny and life cycles, i.e. phenomena occurring on a time-scale going from conception to death (Elsasser 1987). Recently, several worthy contributions have been made in this area (Kupiec 2010; Deacon 2012; Newman 2012; Davies 2013). Approaches based exclusively on stochasticity and natural selection like the ontophylogenesis theory (Kupiec 2010) provide new perspectives; however, they are insufficient to frame a theory of organisms because the molecular events at the core of the ontophylogenesis theory are causally dependent on cell, tissue and organismal contexts and these contexts are not addressed by this approach.

Since the 1970 s, the thermodynamics of dissipative systems provided an opportunity to examine the relevance of self-organizing physical systems to the understanding of the emergence of life, as exemplified by the pioneering work of Prigogine and his school (Nicolis and Prigogine 1977), Kauffman (Kauffman 1993) and others. For instance, Cottrell elegantly highlighted the role of thermodynamics while stressing the need for a biological perspective that would bring to this analysis distinctive biological characteristics such as historicity and purposiveness (Cottrell 1979). 


\section{Theory of Organisms}

Unfortunately, despite these promising beginnings, the development of a theory of organisms has been hindered by the misuse of metaphors borrowed from theories of information (Longo et al. 2012a). To remedy this situation, we are proposing instead explicit principles for the elaboration of a theory of organisms that would make such a theory robust, and open to change when challenged by empirical evidence.

Our analysis purposefully refers to physics, not only because biological theory should not violate physical laws, but also because there is a tradition in organismal biology to use both the similarities and differences between these disciplines to advance biological knowledge. For example, Helmholtz improved the understanding of both the physiology of nerve conduction and muscle metabolism while developing the principle of conservation of energy in physics (Lenoir 1982). An additional example is the current wave of physicists and mathematicians entering the field of Systems Biology while carrying with them the theoretical framework of physics. ${ }^{3}$ Most importantly, biology may make it possible to identify a new physical principle (Moore 2012). Therefore, before stating the proposed principles for a theory of organisms, we will elaborate on some relevant relationships between physics and biology (table 1).

\section{From physics to biology}

\subsection{The impact of physics on biology}

First, we will go over the fundamental role that symmetries and conservation principles play in physical theories, in particular by defining the default state. Then, we will discuss the appearance of new observables, which is related to the concept of emergence, and how these concepts relate to symmetries and conservation principles. We will then posit that biology is characterized by a relentless breaking of symmetries, and propose a theoretical bridge between physics and biology which we are calling extended criticality. Extended criticality provides the theoretical frame for a nonconservative default state. The latter entails an intrinsic source of variation, which is a necessary concept in evolutionary biology together with a principle of non-identical iteration of morphogenetic processes.

\footnotetext{
${ }^{3}$ However, applying existing physical theories directly to biology might be misleading. For example, in numerous studies the use of the concepts of energy and temperature stems from a fruitful mathematical analogy with statistical mechanics. However, the analog of temperature and energy in models of interacting birds in a flock is not and cannot be expressed in the proper physical units (Joules for energy and Kelvin for temperature); as a result, these applications lack a theoretical justification for this energy to be conservative which is a crucial invariant property for statistical physics. A specific example of why any scientific theory developed within a specific domain cannot be directly applied to another domain is given in Longo et al. (2012a) about the application of the concept of information in biology.
}

\subsection{Default states and symmetries in physics}

Since Galileo and Descartes, physicists made the default state of inert matter explicit: this is inertia, an instance of fundamental conservation laws. ${ }^{4}$ Briefly, if no cause (force) modifies the properties of an object, the object conserves its properties. ${ }^{5}$ In short, a default state is what happens when nothing is done to the object in question. Three centuries later, E Noether's theorems provided a deeper understanding for this default state by mathematically justifying conservation properties of energy and momenta in terms of symmetries in the state equations (van Fraassen 1989). Ever since, symmetries (and their breaking) acquired an even more fundamental role in physics (table 1). We are referring to the notion of 'symmetries' in the broad sense given to it by modern physics, as transformations preserving the key invariants observed and proposed by the intended theory. For example, Noether's theorem enables us to understand the conservation of energy and momentum on the basis of time and space symmetries of fundamental equations, respectively. In short, the conservation of these quantities is grounded on the idea that the 'laws' of physics are the same at different positions and times. However, the notion of symmetries is as old as Archimedes' principles as exemplified by the lack of movement of a scale with equal and symmetric weights on each arm. Moreover, physicists have proposed the existence of a particle for symmetry reasons; the existence of antimatter was proposed this way. In biology, instead, the types of symmetries usually referred to are a subset within the broad category of symmetries in mathematics; for example, symmetry with respect to an axis on a plane. They represent specific and simple cases of transformation (a space rotation, for example) preserving the properties of the geometric structure under examination.

\subsection{Emergence in physics and in biology}

The construction of theories in physics relies on mathematical symmetries. There is no obvious continuity between theories when they are based on different symmetries. For example, in classical mechanics there is no time arrow (phenomena are reversible) whereas in thermodynamics, like in biology, time is oriented (phenomena are irreversible) ${ }^{6}$ (table 1).

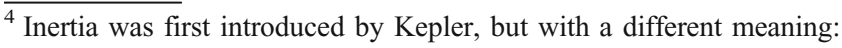
the default state was rest (quiescence), and thus, it could not explain why a planet keeps orbit without being pushed by some agency.

${ }^{5}$ In the context of Galilean relativity, speeds are arbitrary or relative, and entail a change in position; therefore, position cannot be conserved. The speed of an isolated object is conserved.

${ }^{6}$ For example, irreversibility means that we can tell when a movie is being played forward or backward.
} 
Giuseppe Longo et al.

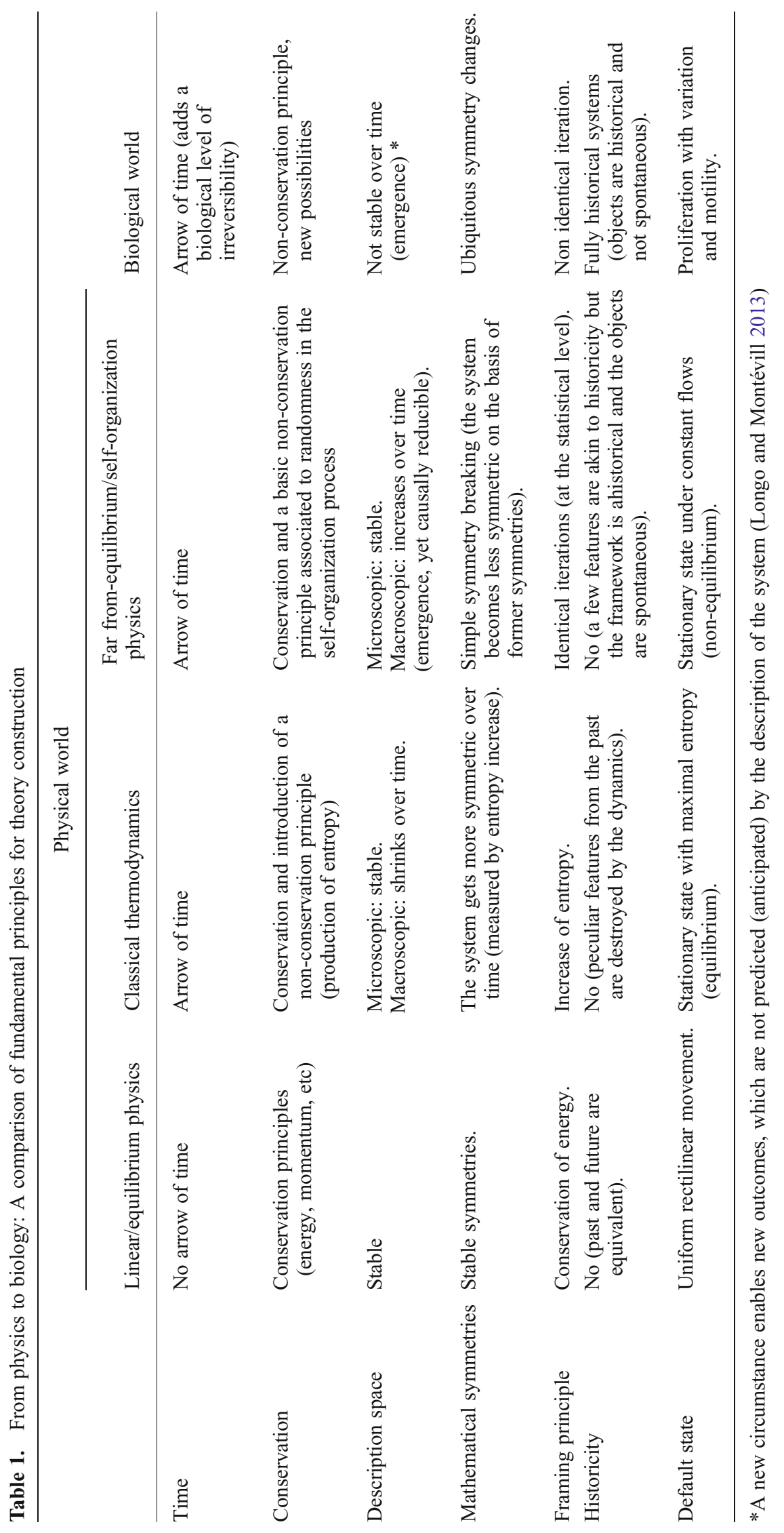




\section{Theory of Organisms}

Emergence is the appearance of a new observable that cannot be derived from the root theory. The transition from water molecules to a liquid is a relevant example of emergence of new properties, such as fluidity and incompressibility. These novelties force a radical change of mathematical symmetries and thus of theories from QM to hydrodynamics (HD). Similarly, at its inception, TD disregarded gas particles and their classical trajectories, and focused instead on macroscopic observables, such as temperature, volume and pressure. Later on, Boltzmann unified TD to the Newton-Laplace theory of trajectories of gas particles by adding the hypothesis of 'molecular chaos', a strong, limit property, based on a fundamental symmetry. ${ }^{7}$ As a result, the theoretical symmetries were totally changed. For example, time becomes irreversible (table 1). In both cases a new unifying theory, including theoretical symmetry changes, is needed. Recapitulating, in physics a theory is ruled by its mathematical symmetries, which in turn determine its proper observables (table 1). Conversely, the appearance of new observables forces a change of theory.

A biological example of emergent phenomena is the advent of novelties such as ears and hearing which evolved from the double jaw bones of some vertebrates. These evolutionary processes imply a change of observables and, thus, of theoretical symmetries (Longo and Montévil 2014). In the previous examples from physics, changes of observables and symmetries required a change of theory. In contrast, Darwin proposed a global theory of evolutionary dynamics, one with little mathematics and with no explicit theoretical symmetries, yet based on principles that allow us to understand changes like the generation of new organs and functions ${ }^{8}$ and of new phenotypes. Implicitly, Darwin's is a theory about symmetry changes; that is, a theory of the changes of biological organization along phylogenesis. Symmetry changes are also relevant for ontogenesis, as exemplified from the generation of a metazoa from a single cell. We thus propose principles for a theory of organisms in which symmetry changes are incessant, and occur in an 'extended critical interval' (Bailly and Longo 2011; Longo and Montévil 2014) (see below). Unification between ontogenesis, and phylogenesis will require a closer examination of criticality.

\subsection{From criticality to extended criticality}

In the 1980s, theoretical biologists became interested in the physics of criticality where symmetry breaking is a central

\footnotetext{
${ }^{7}$ More precisely, this unification uses the notion of ergodicity. The ergodic hypothesis states that a particle spends time in regions of the phase space of same energy proportionally to their volume, which is a new symmetry of the phase-space.

${ }^{8}$ We use a broad definition of function: the physiologic activity of an organ or part.
}

concept (Bak and Sneppen 1993; Kauffman 1993). Moreover, along critical transitions, the 'local structure' is correlated to the 'global' one, a phenomenon which echoes the coherence of organisms. Typically, at critical transitions, correlation lengths ${ }^{9}$ tend to infinity and the very object of investigation changes, say, from vapour to a snowflake. This transition happens at a point which marks the passage from one object to another and from one symmetry to another. Within a given theoretical framework, renormalization methods allow the representation of the critical transition to a 'new object' by a class of models parameterized by the scale $^{10}$ (table 1). In biology, various phenomena are analysed explicitly as critical transitions in the physical sense, such as the formation of a coherent depolarization of thousands of mitochondria inside cardiomyocytes (Aon et al. 2004), the critical dynamics of the transcriptome (Nykter et al. 2008) and the coherent activities of neurons (Werner 2007) (see Longo and Montévil (2014) and Mora and Bialek (2011) for additional examples).

From the view point of extended critical transitions an organism is understood as being in a permanent transition with all the main signatures of criticality, such as changes of symmetries, the constant reconstruction of correlation lengths and the formation of a new global structure (Bailly and Longo 2011; Longo and Montévil 2014) (table 1). Thus, phylogenetic and ontogenetic trajectories involve 'cascades of symmetry changes' which contribute to the historicity of phylogeny and ontogeny by generating biological variability and anatomical and functional diversity (Longo et al. 2012b; Longo and Montévil 2014). This is the first of the points we wish to make regarding the unity of these two theories addressing life at different time-scales.

The theory of critical phase transitions is relevant to our proposal because this theory is concerned with the formation of new objects and symmetries at and beyond the critical transition point (figure 1). Here emergence of a new object is mathematically treated as a point-wise transition at the critical point (Longo and Montévil 2014). Extended critical transitions, instead, span a non-trivial interval such as an organism's lifetime. In this context, an organism continually undergoes critical transitions, whereby both the objects and the symmetries change. The organism and its components are permanently reconstructed with variations. ${ }^{11}$ This represents a sharp departure from physics where a radical change of symmetry implies a change of theory. So far, only a single

\footnotetext{
${ }^{9}$ Correlation length: distance at which different parts still influence each other.

${ }^{10}$ The renormalization methods provide refined mathematical techniques to deal with the situation at a symmetry change (Lesne 1998).

${ }^{11}$ This situation is very different from the physical notion which involves a single point and a single symmetry change. At the mathematical limit, this may be viewed as a dense set of critical transitions in the intended interval (Longo and Montévil 2014)
} 


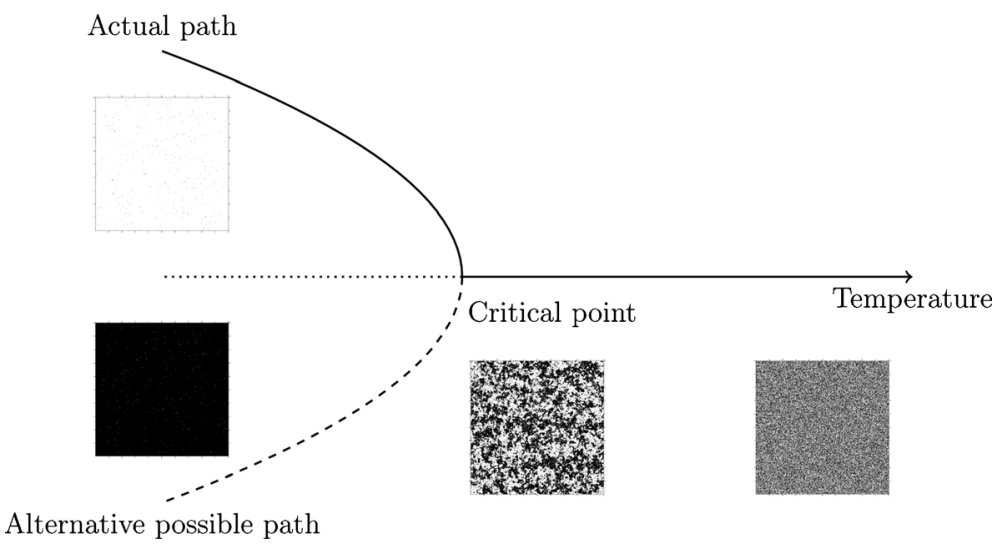

Figure 1. This figure represents a simulation of a phase transition, which is a simple model of magnets: the Ising model. The system is a grid where each location can be either 1 (white) or -1 (black). At high temperatures, the system is disordered, and is a uniform random mix of black and white; as such its macroscopic description does not change if we swap black and white: it is symmetric and the global magnetization is 0 . At low temperatures, the system is magnetized, and is either dominated by black or white; the magnetization has become a new relevant global observable and the symmetry is broken. At the critical point, the point of transition between these two states, the system has a global behaviour with patterns at all scales.

transition like the one at the critical point can be accommodated into a single theory. In summary, the passage from theories of the inert to a theory of organisms must accommodate continual symmetry changes within one theoretical frame. Extended criticality is an attempt in this direction.

\subsection{Physical systems at equilibrium, far-from-equilibrium and organisms}

Physical systems at equilibrium are fully described by conservation properties and the associated equational symmetries. In contrast, far-from-equilibrium dissipative physical systems like flames, micelles and Bénard cells ${ }^{12}$ are an organization of flows of energy and/or matter. In a mathematical sense, flows and boundary conditions fully determine and thus causally trigger and maintain the structure of any such system (Nicolis and Prigogine 1977). Within this theory, the quantity which allows the mathematical analysis of the system is entropy production, that is, the energy dispersal rate instead of energy conservation. This analysis is based on a non-conservative quantity, i.e. entropy increase associated to time irreversibility. When the flows of energy, matter and entropy are constant, these systems are called stationary. However, not all the mathematical symmetries of equilibrium are still applicable. Instead, the balance equations of the flows provide more suitable tools for the analysis

\footnotetext{
$\overline{12}$ Bénard cells are one of the simplest types of self-organization of matter generated by natural convection: regular patterns of liquid movement occur in a layer of fluid heated from below.
}

of stationary systems than those equations of equilibrium (Cottrell 1979; Nicolis and Prigogine 1977). In this instance, physics approximates biology because there is no such thing as an organism at thermodynamic equilibrium. Organisms use flows but are not a consequence of flows (table 1). Next, we further elaborate on this distinction.

Far-from-equilibrium physical systems are understood by the analysis of their instantaneous flows. Indeed, the shape of a flame can be calculated from the flows of energy and matter that go through it, whereas the shape of an organism cannot. Farfrom-equilibrium systems are ahistorical ${ }^{13}$ because they appear spontaneously and can be analysed independently. In contrast, organisms are not spontaneous but historical, meaning that they are a consequence of the reproductive activity of a pre-existing organism. Organization cannot be deduced from flows operating within and upon organisms; instead, understanding biological organization requires a historical analysis. This perspective reinforces Dobzhansky's assertion that 'nothing in biology makes sense except in the light of evolution' given that evolution is precisely historical. Historicity is thus the second point we stress in this quest for unification between the theories of evolution and of organisms.

In an organism, each cell division changes local symmetries because each of those divisions forces new local and potentially global correlations. These changes yield variability and adaptability to organisms. In the context of evolution, the advent of new functions and organs are additional

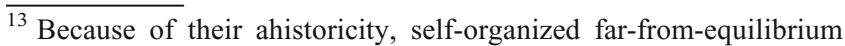
physical systems are used as a paradigm for the origin of life, a subject that is not addressed herein.
} 


\section{Theory of Organisms}

examples of symmetry changes. In our approach, reproduction with modification involves 'symmetry changes' and may be viewed as multiple critical transitions which span the irreversible time of both phylogenesis and ontogenesis. This is the third common point towards the unification of the theories addressing these two different time-scales.

\section{Foundations for a Theory of Organisms}

We propose two founding principles: (1) the default state of cells, meaning proliferation with variation and motility, and (2) the framing principle of non-identical iterations of a morphogenetic process. These principles take place in the context of extended critical transitions.

\subsection{The default state in biology: A nexus between the theories of evolution and of organisms}

In order to provide a theoretical transition between physics and biology, we will define a default state which is a limit case. Inertial movement as uniform rectilinear movement is a limit state and physicists made all physical movements intelligible as departures from it. By describing this default state, Galileo could focus on the analysis of the forces constraining it, such as gravity and friction.

The 'unconstrained' condition proper to the biological default state requires adequate physical conditions, such as specific intervals of temperature, pressure and $\mathrm{pH}$. Sufficient nutrients provide a flow of energy and matter canalized through metabolic processes. In these unrestrained, limit conditions, cells constitutively exert their default state, i.e. proliferation with variation plus motility (table 1). The default state should not be conflated with conditions necessary for life. For example, metabolism is necessary for cells to be alive given that metabolism happens regardless of whether cells are proliferating or quiescent, moving or immobile ('metabolism happens, whatever happens'). In contrast, a default state is what happens when nothing is done to the object or system ('a default state happens when nothing happens to prevent it'). Consequently, the nature of what can be done to the system is defined by the theory, like the concept of force in classical mechanics; according to Newton's first law force modifies (accelerates) inertial movement. Thus, the analysis of the constraints to proliferation with variation and motility is fundamental to the intelligibility of organismal biology. This is equivalent to the role of

\footnotetext{
${ }^{14}$ The term constraint has been used in various contexts. The concept used herein is (i) constraint is something that remains invariant with respect to the duration of the process being constrained, (ii) a constraint changes the process being constrained (Montévil and Mossio 2015). Additionally, like for the mechanical default state (inertia) a constraint acts on the biological default state. In biological systems, constraints enable the emergence of new processes and phenomena.
}

Newton's laws for understanding mechanics. It should be remembered that Newton posed his laws 'axiomatically,' as core principles of his theory. ${ }^{14}$

4.1.1 Proliferation with variation: Darwin explicitly stated '...There is no exception to the rule that every organic being naturally increases at so high a rate, that, if not destroyed, the earth would soon be covered by the progeny of a single pair' (Darwin 1859). Reproduction obligatorily involves 'modification' (descent with modification, in Darwin's words). Reproduction with variation is intrinsic to organisms regardless of whether they are unicellular or multicellular (Sonnenschein and Soto 1999; Soto and Sonnenschein 2011). Darwin's narrative implies that reproduction with variation is a default state and he describes it as a limit case. This is the third, and probably most important, point in common between the theories of evolution and organisms.

The default state of proliferation applies to the first common ancestor, i.e. the cell from which all organisms arose (figure 2). In fact, microbiologists consider axiomatic that proliferation is the default state of prokaryotes and unicellular eukaryotes (Luria 1975). On the contrary, despite lacking evidence, there had been a consensus among biologists that consider quiescence as the default state of cells in multicellular organisms (Alberts et al. 2008; Alberts 2010). We posited, instead, that proliferation was retained as the default state with the advent of metaphyta and metazoa. This conclusion is supported by the conservation of the cell cycle components throughout eukaryotes (Sonnenschein and Soto 1999) and by experimental evidence (Soto and Sonnenschein 1985; Sonnenschein et al. 1996; Sonnenschein and Soto 1999; Ying et al. 2008; Leitch et al. 2010). Additionally, the default state of proliferation has been adopted advantageously as a fundamental principle in theories of carcinogenesis and of development (Sonnenschein and Soto 1999; Soto and Sonnenschein 2010; Minelli 2011).

Variation (as in proliferation with variation) should be understood as symmetry changes; each cell division generates variations that correspond to symmetry changes associated to critical transitions. How does intrinsic variation manifest itself? One obvious example is the unequal distribution of macromolecules and organelles during cell division (Huh and Paulsson 2011); another is stochastic gene expression (Kupiec 1983; Taniguchi et al. 2010; Tyagi 2010; Marinov et al. 2014). Additional variation is due to somatic mutations and aneuploidy, events described among cells of normal mammalian kidneys and brains (Martin et al. 1996; Rehen et al. 2001).

4.1.2 Motility: Motility encompasses intracellular, cellular, tissue and organismic movements (Stebbings 2001). The 
Giuseppe Longo et al.

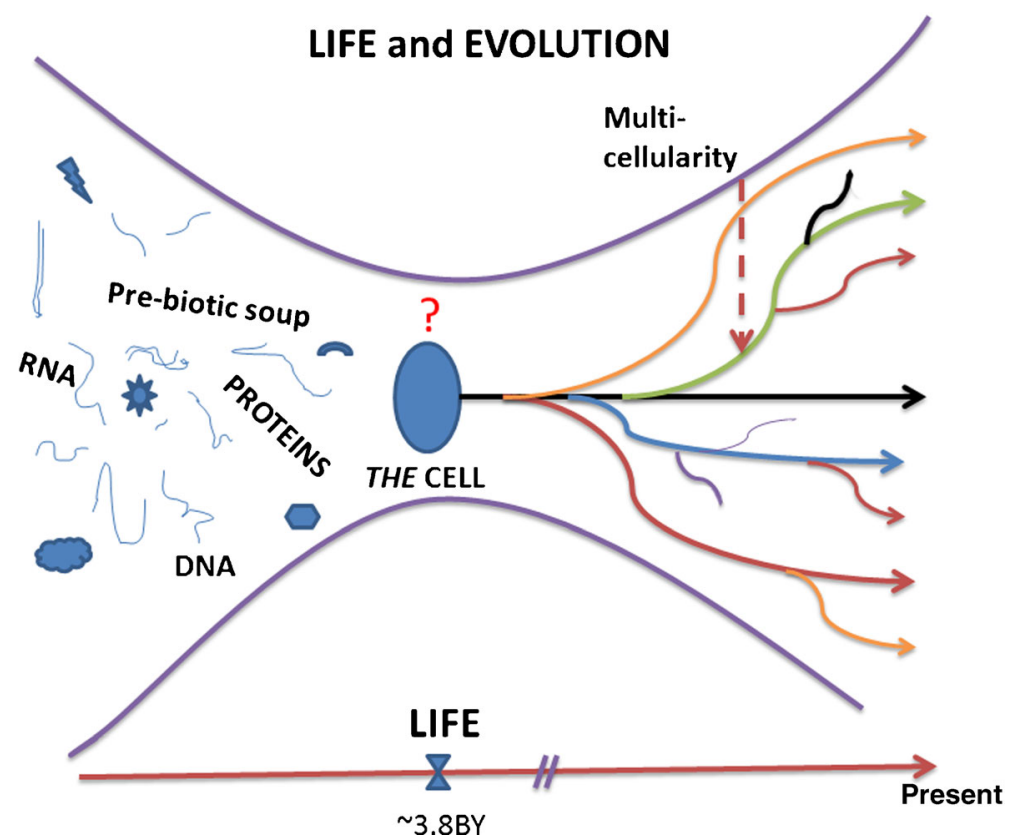

Figure 2. The emergence of living organisms and their further evolution, as we know it, implies that the default state of the first cell (i.e. 'THE CELL') and those of their daughters must have been proliferation-with-variation and motility. The question mark indicates that the process that generated THE CELL from a pre-biotic soup is unknown.

evolution of the cytoskeleton protein actin, which is believed to have been present in all early organisms, supports the concept that motility, like proliferation with variation, is the default state of all cells (Buss 1987; Sonnenschein and Soto 1999). From chemotaxis to swimming, motility immediately suggests the idea of agency. ${ }^{15}$ Agency has dominated the debate on the differences between inert and living matter for most of the 19th century. Motility suggests a comparison with classical physics because both involve trajectories in space. In physics, an external force is required to obtain a change of inertial movement, interpreted as a conservation property grounded on theoretical symmetries. In contrast, a cell or an organism will spontaneously move by using forces and flows of energy and matter. Cells do not require external stimuli to move. Movement along a direction represents a symmetry change, and corresponds to a non-conservation property as it is not inertial. Moreover, these actions are accompanied by critical transitions ( Werner 2007; Cardamone et al. 2011).

Motility should not exclude movements other than locomotion. Plants may be attached to the ground by their roots, but they can generate movement of their parts, for example by growing towards a source of light. Flowers and leaves

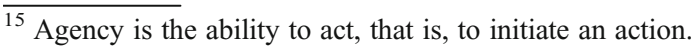

open and close in response to light (van Doorn and van Meeteren 2003), and like animal cells, can move organelles using actin and myosin (Ueda et al. 2010).

\subsection{Default state and constraints}

During early development, increases in the size of an organism occur by generation of new cells and production of extracellular matrix. Cell proliferation is the result of the default state and is constrained. Organ shape is a result of the cells' default state plus physical constraints, like gravity, and those ones created by the living organism, like muscular tension.

Inert matter requires causes to change states or properties. The causal structure of a physical process is determined mathematically by a set of equations justified by symmetries, such as equilibria. For example, Newton's equations relate forces to their effects, i.e. as causes of acceleration at equilibrium. In physics, causes and constraints on the default state are synonymous. Organisms, instead, are agentive and thus capable of initiating activity by themselves. The default state is a cause in biology; by contrast, anything that affects the default state is a constraint. For example, gravity becomes a constraint for evolution and embryogenesis, and not a cause of biological dynamics. Gravity influences numerous if not all biological processes and it is a main determinant of 


\section{Theory of Organisms}

morphogenesis as exemplified in the formation of the anteroposterior axis in chick embryos. However, the formation of this axis is not a consequence of gravity, but of the activity of cells under the constraint of gravity: in our theoretical frame and language, gravity is a constraint and not a cause. In sum, biological dynamics is grounded on the default state, that is, proliferation with variation and motility.

Constraints narrow down the range of 'possibles'. For example, fibroblasts removed from subcutaneous tissues of an animal have the same size when dissociated and explanted into a culture dish. Soon after, their size varies over a wide range ( Rubin and Hatie 1968; Rubin 1988). Constraints also enable other 'possibles.' The bottom of a cell culture dish prevents displacement in that direction reducing the possible movements, but at the same time this constraint enables cells to crawl along a plastic surface. In a multicellular organism, other constraints to the default state are relevant. Mechanical constraints limit proliferation, variation and motility, like when cells compress each other; they adhere to other cells, and stretch each other. Fibers in the extracellular matrix apply tensile forces on structures allowing movement in certain directions and constraining it in others (Barnes et al. 2014). Sequential smooth muscle differentiation exerts compressive stress on the endoderm and mesenchyme of the small intestine, causing buckling and folding which leads to the formation of villi (Shyer et al. 2013). Muscle contraction shapes the bones to which they are attached by inducing tissue accrual on the side of tension (Muller 2003; Rot-Nikcevic et al. 2007), and chemical interactions may also constrain the default state, as when serum albumin restricts proliferation of estrogen sensitive cells, and estrogen cancels the action of this constraint (Sonnenschein et al. 1996).

We posit that organisms do not have stable symmetries that would allow us to spell out the actual phylogenetic and ontogenetic trajectories that they follow because such trajectories depend on the history and random changes of symmetries of the objects considered, organisms in this case (Longo and Montévil 2014). The default state of proliferation with variation corresponds to Darwin's key idea of evolution being 'descent with modification', on which selection operates.

In contrast to physics where conservation principles frame the theories, in biology, the default state of proliferation with variation is a non-conservation principle (Longo et al. 2012b; Longo and Montévil 2013). This rationale does not conflict with physical principles as it concerns new observables, i.e. phenotypes. In fact, proper principles and observables are being added at different and interacting levels of biological organization and determination. Thus, the theory of organisms that we envisage becomes a compatible extension of physical theories.

Finally, the recent phylogenetic history of every cell is the proliferation of its parental cell. On a far longer temporal scale unicellular ancestors of cells of multicellular organisms are assumed to have had proliferation as their default state. Thus, it is the shift from proliferation with variation and motility to quiescence, which should be explained, instead of being assumed. Cell proliferation is achieved by the execution of a cell cycle process, which does not stop until two quite similar but non-identical cells are formed. The cell cycle is a representation of enzymatic reactions and physical processes leading to the duplication of the cell components including DNA, and the faithful separation of two daughter cells. Textbooks use the metaphor of the cycle operating like a dishwasher performing a series of stereotyped tasks (Alberts et al. 1994). If quiescence were its default state, it would be difficult to activate the 'cell cycle machine' by organizing the cell cycle components which entail a very complex network of constraints. Instead, when proliferation is the default state, it becomes easier to prevent complex machinery from functioning: a simple mechanical or chemical constraint may suffice. For example, a switch will do it for an engine and an inhibitor will do it for a cell. To sum the situation up, the difference between proliferation or quiescence as a default state corresponds to the difference between preventing something complex from happening and causing it to happen. The latter is much harder to conceive, explain and realize.

\subsection{The framing principle of biological morphogenesis}

Generating phenotypes from a single cell, be it LUCA (Last Common Universal Ancestor) (Steele and Penny 2010) or a zygote, is an essential component of phylogenesis and ontogenesis. Organisms, be they unicellular or multicellular, are a consequence of the inherent variability generated by proliferation, motility (Sonnenschein and Soto 1999) and selforganization (Mossio and Moreno 2010), all of which operate within the framing principle we propose: life phenomena are non-identical iterations of a morphogenetic process by which organization is iterated and maintained (table 1). For example, branching morphogenesis is a ubiquitous process that generates a repetitive, yet non-identical pattern whereby length of branches and branching angles vary (figure 3).

The framing principle cannot be derived from the default state alone. It may be instantiated as autopoiesis ${ }^{16}$ or more generally, closure to which variation is added, given that living autopoietic processes require permanent changes in their constructing and reconstructing activities (Montévil and Mossio 2015). Far-from-equilibrium physical processes have autopoietic characteristics by iterating shapes and physical

\footnotetext{
${ }^{16}$ Autopoietic organization is defined as a unity by a network of productions of components which (i) participate recursively in the same network of productions of components which produced these components, and (ii) realize the network of productions as a unity in the space in which the components exist (Varela et al. 1974).
} 
Giuseppe Longo et al.
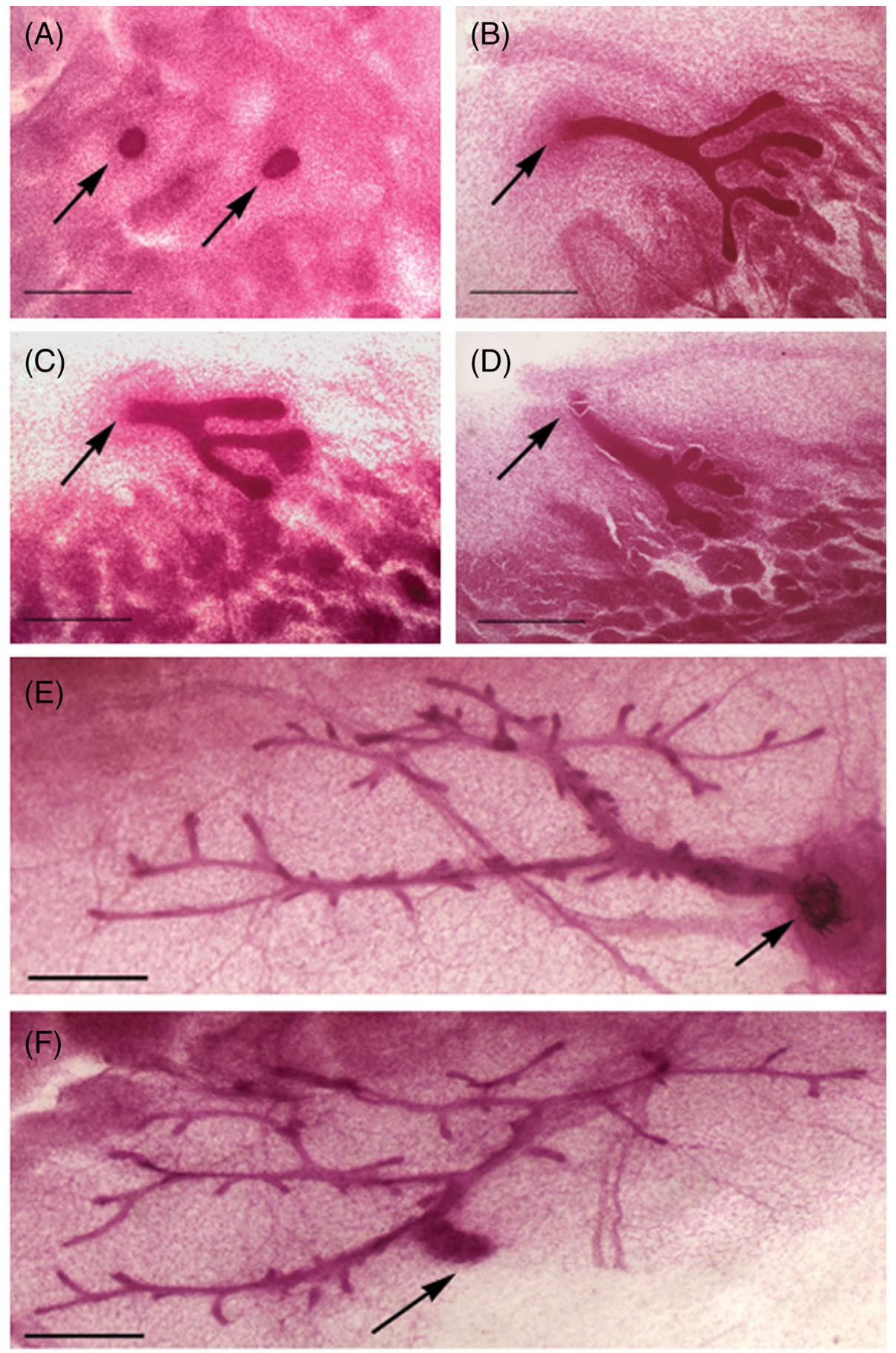

Figure 3. Ductal development in whole mounts of mammary glands from C57B16 mice illustrates the principle of never identical iterations on the branching pattern of a ductal system. In Panel A, the arrows point to two mammary gland epithelial structures (buds) at day 15 of embryonic life (E15); the ductal tree emerges from the growth of these buds. Panels B to D show the ductal tree at E19. Arrows indicate the origin of the ductal tree. Scale bars indicate $100 \mu \mathrm{m}$. Panels $\mathbf{E}$ and $\mathbf{F}$ show mammary trees at post-natal day 21 . Scale bars indicate $500 \mu \mathrm{m}$.

structures along optimal trajectories. Flames, micelles and Bénard cells remained unchanged over the last 4 billion years while evolution generated diverse living forms from LUCA up to the reader of this page. In our approach, we 


\section{Theory of Organisms}

expand the notion of autopoiesis by including in it the concept of variability. The latter is expressed as theoretical symmetry changes. By these means we go beyond properties that could be purely physical since autopoiesis would be enriched by biological variability and historicity, both associated with symmetry changes.

\subsection{Complexity versus organization}

Oftentimes, organization ${ }^{17}$ and complexity are considered synonyms. However, while complexity is mostly meant to span the material world, including inert and living matter, organization should be viewed as an exclusive attribute of life and of machines invented by humans. Complexity and organization ought to be explicitly defined and distinguished. We posit that phenotypic complexity is a quantifiable characteristic of static structures as exemplified by the anatomy of an organism. Complexity can be quantified by enumerating its components such as the number of cell types, tissues, organs, organ-connected components, connections and nodes within networks, fractal dimensions of cells and organs, etc. (Bailly and Longo 2009; Bizzarri et al. 2011; Longo and Montévil 2014). From the perspective of the framing principle, instead, organization refers to a dynamic state of interdependence of levels that includes both structures and functions as well as integration and regulation. Organization cannot happen without complexity, but organization is not reducible to complexity.

Carcinogenesis illustrates how complexity and organization are not equivalent. For instance, in the mammary gland, precancerous lesions like ductal carcinoma in situ are more complex than normal ducts. This lesion appears as a partial occlusion of ducts which results in more than a single lumen. Multiple lumena indicate higher measurable topological complexity (figure 4). Moreover, the epithelium-stroma interface has a higher fractal dimension than that of their normal counterparts (Bizzarri et al. 2011). ${ }^{18}$ However, a cancerous tissue is less organized in the sense that it does not adequately perform the function of the normal tissue of origin. For example, a blocked duct interferes with excretory function. Additionally, cells within cancer tissues may not

\footnotetext{
${ }^{17}$ The term organization appeared in the early 15 th century, in both English and French; it represents a conflation of 'to construct' and 'organ,' the musical instrument. Organization became associated with the meaning of organized living beings in the 18th century. In our narrative, organization is compatible with the notion of 'organizational closure'. The latter is a 'distinct level of causation, operating in addition to physical laws, generated by the action of material structures acting as constraints' (Mossio and Moreno 2010).

18 'Cells within a cancer' is not synonymous with 'cancer cells'. When considering that cancer is a tissue-based disease the phenotype of individual cells within the cancer tissue is determined by the tissue. For a detailed explanation, see (Soto and Sonnenschein 2011)
}

perform the functions of the cells within the normal tissue of origin. This combination of higher complexity and lower organization represents a true hallmark of cancer.

\section{Implications for biological research}

What are the benefits of adopting the principles for a theory of organisms delineated above? First, the proposed principles would help to move away from operational definitions. From the notion of gene to that of growth factor, the use of operational definitions has resulted in contradictions and ambiguities that hinder the establishment of general and stable concepts (Moss 2003; Sonnenschein and Soto 1999). Additionally, the proposed principles enable alternative explanations to the mechanistic ones inherent to the molecular biology revolution. The latter do not represent an explicit theoretical frame, but mostly refer metaphorically to common sense notions, such as 'information' and 'program'. The use of these metaphors forces explanations to be molecular and to follow predictable causal chains (Longo et al. 2012a). Instead, by insisting on the search of constraints to the default state, multilevel biomechanical and bioelectrical explanations become as legitimate as molecular ones.

Second, our principles radically change both observables and determination vis-à-vis the theoretical frames proposed by physical theories. Such a change enables us to anchor reasoning and modelization on robust biological principles. Indeed, as implied by Turing, there is an epistemological gap between modelization and imitation (Turing 1950, 1952). While the former is based on a theory concerning the object of knowledge, the latter is not. For example, individuation becomes the result of non-identical morphogenetic iterations. This principle excludes a Platonic conception of ideal or perfect organs or structures which would be determined as an optimal solution of an equation.

Third, the principles we propose enable the construction and discussion of mathematical models on the bases of biologically relevant assumptions. For example, in ecology the commonly used equations addressing population size are not theory-based. Taking into consideration the default state and the notion of constraint it is feasible to obtain theoretically meaningful equations in which the food term is not a cause per se: this constraint becomes relevant only when there is scarcity of food. In an ecological context there are numerous parameters that enable the population to grow in number, however, those parameters actually play a role on growth (i.e. the growth rate) only when they are limiting the increase of the population. Food superabundance will not make the population grow faster.

Fourth, we are proposing an alternative to the use of the notions of program, information, and signal specificity in 


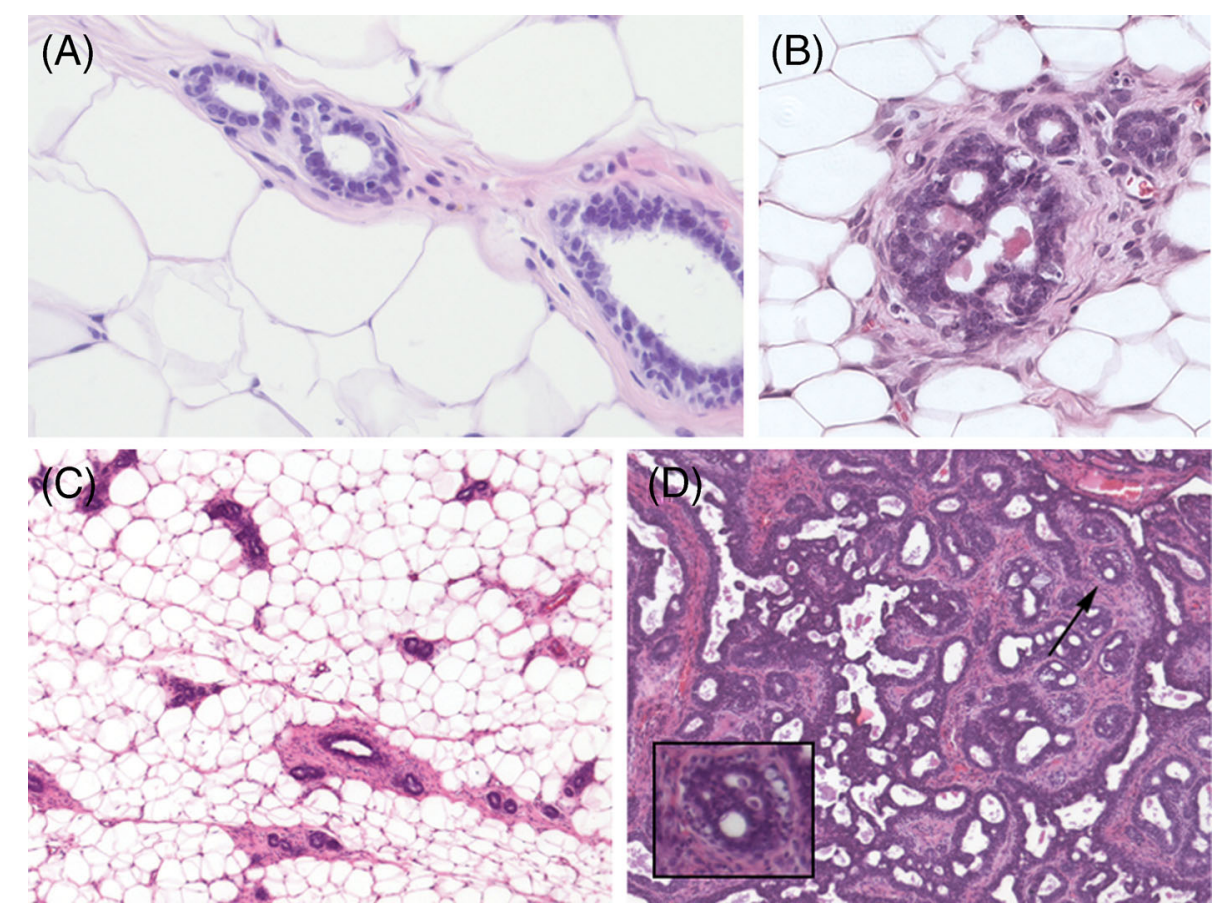

Figure 4. Complexity versus organization. Panel A shows cross-sections of normal mammary gland ducts. Panel B shows a cross-section of a ductal carcinoma in situ. Note that while normal ducts have a single lumen, carcinoma in situ has multiple ones, thus showing higher topological complexity than normal ducts. Panel $\mathbf{C}$ shows various cross-sections of normal ducts, and Panel $\mathbf{D}$ shows a section of a large carcinoma tumour. The arrow points to the area that is magnified in the inset. The tumour contains many structures similar to the carcinomas in situ.

biology. In particular, biological variation is not noise as in those information-based theories (Huang 2009). Rather than as a priori determination as presupposed by the notion of developmental program, the two proposed principles lead to a conception of biological specificity that is defined directly with respect to the trajectory of organisms and their cells in time and space as a cascade of symmetry changes.

\section{Conclusions}

Research on organismal biology is being conducted in the absence of a global theory. Instead, its conceptual framework is loosely based on the mathematical theories of information and on operational definitions. This combination of notions has generated contradictions and hindered progress. In spite of a few successful outcomes, the application of physical principles without proper analysis of the differences between biological and physical situations has also contributed to the current crisis. Our analysis of the differences between the physics of inanimate and living matter provides a sound perspective for the construction of a much needed theory of organisms while providing founding principles adequate for framing experiments and mathematical modelling.

\section{Acknowledgements}

This work was conducted as part of the research project 'Addressing biological organization in the post-genomic era', which is supported by the International Blaise Pascal Chairs, Region Ile de France. AMS is the incumbent Blaise Pascal Chair of Biology at the École Normale Supérieure, and GL is the Chair's host. Additional support was provided by grants from the Avon Foundation, the Italian Space Agency and the National Institute of Environmental Health Sciences of the NIH (ES08314 and U01-ES020888). The content does not necessarily represent the official views of the funding agencies including the National Institute of Environmental Health Sciences or the National Institutes of Health. MM was supported by a grant from the Région Îlede-France, DIM 'Problématiques transversales aux Systèmes Complexes'. CS is a Fellow of the Institut d' Études Avancées de Nantes. GL's work is part of the project 'Les 


\section{Theory of Organisms}

lois des Dieux, des hommes et de la nature' in that Institute. The authors are grateful to Paul-Antoine Miquel, Matteo Mossio, Andrew Moore, Cheryl Schaeberle and Michael Sweeney for their critical input. The authors have no competing financial interests to declare.

\section{References}

Alberts B 2010 Model organisms and human health. Science 3301724 Alberts B, Bray D, Lewis JG, Raff M, Roberts K and Watson JD 1994 Molecular biology of the cell (New York, NY: Garland Publishing Inc.)

Alberts B, Johnson A, Lewis J, Raff M, Roberts K and Walter P 2008 Molecular biology of the cell (London: Garland Science)

Aon MA, Cortassa S and O'Rourke B 2004 Percolation and criticality in a mitochondrial network. Proc. Natl. Acad. Sci. USA $1014447-4452$

Bailly F and Longo G 2009 Biological organization and anti-entropy. J. Biol. Syst. 17 63-96

Bailly F and Longo G 2011 Mathematics and natural sciences: the physical singularity of life (London: Imperial College Press)

Bak P and Sneppen K 1993 Punctuated equilibrium and criticality in a simple model of evolution. Phys. Rev. Lett. 71 4083-4086

Barnes C, Speroni L, Quinn K, Montevil M, Saetzler K, BodeAnimashaun G, McKerr G, Georgakoudi I, et al. 2014 From single cells to tissues: interactions between the matrix and human breast cells in real time. PLoS One 9 e93325

Bizzarri M, Giuliani A, Cucina A, D’Anselmi F, Soto AM and Sonnenschein C 2011 Fractal analysis in a systems biology approach to cancer. Semin. Cancer Biol. 21 175-182

Buss LW 1987 The evolution of individuality (Princeton, NJ: Princeton University Press)

Cardamone L, Laio A, Torre V, Shahapure R and DeSimone A 2011 Cytoskeletal actin networks in motile cells are critically self-organized systems synchronized by mechanical interactions. Proc. Natl. Acad. Sci. USA 108 13978-13983

Cottrell A 1979 The natural philosophy of engines. Contemp. Phys. $201-10$

Darwin C 1859 On the origin of species (London: Clowes and Sons)

Davies J A 2013 Mechanisms of morphogenesis (Elsevier Academic Press)

Deacon T 2012 Incomplete nature: how mind emerged from matter (New York: Norton)

Elsasser WM 1987 Reflections on a theory of organisms (Quebec: Orbis Publishing)

Huang S 2009 Non-genetic heterogeneity of cells in development: more than just noise. Development 136 3853-3862

Huh D and Paulsson J 2011 Non-genetic heterogeneity from stochastic partitioning at cell division. Nat. Genet. 43 95-100

Jacob F 1974 The logic of life (New York, NY: Pantheon Books)

Kauffman SA 1993 The origins of order (Oxford: Oxford University Press)

Kupiec JJ 1983 A probabilistic theory for cell differentiation, embryonic mortality and DNA C-value paradox. Specul. Sci. Technol. 6 471-478
Kupiec JJ 2010 On the lack of specificity of proteins and its consequences for a theory of biological organization. Prog. Biophys. Mol. Biol. 102 45-52

Leitch HG, Blair K, Mansfield W, Ayetey H, Humphreys P, Nichols J, Surani MA and Smith A 2010 Embryonic germ cells from mice and rats exhibit properties consistent with a generic pluripotent ground state. Development 137 2279-2287

Lenoir T 1982 The strategy of life: teleology and mechanics in nineteenth-century biology (Dordrecht, Holland: D. Reidel Publishing)

Lesne A 1998 Renormalization methods: critical phenomena, chaos, fractal structures (New York: Wiley)

Longo G and Montévil M 2012 The inert vs. the living state of matter: extended criticality, time geometry, anti-entropy - an overview. Front. Physiol. 339

Longo G and Montévil M 2013 Extended criticality, phase spaces and enablement in biology. Chaos, Solitons Fractals 55 64-79

Longo G and Montévil M 2014 Perspectives on organisms: biological time, symmetries and singularities (Berlin: Springer)

Longo G, Miquel P-A, Sonnenschein C and Soto AM 2012a Is information a proper observable for biological organization? Prog. Biophys. Mol. Biol. 109 108-114

Longo G, Montévil M, and Kauffman S 2012b No entailing laws, but enablement in the evolution of the biosphere; in Genetic and evolutionary computation conference (Philadelphia: ACM)

Luria SE 197536 lectures in biology (Cambridge: MIT Press)

Marinov GK, Williams BA, McCue K, Schroth GP, Gertz J, Myers RM and Wold BJ 2014 From single-cell to cell-pool transcriptomes: stochasticity in gene expression and RNA splicing. Genome Res. 24 496-510

Martin GM, Ogburn CE, Colgin LM, Gown AM, Edland SD and Monnat RJ Jr 1996 Somatic mutations are frequent and increase with age in human kidney epithelial cells. Hum. Mol. Genet. 5 215-221

Minelli A 2011 Development, an open-ended segment of life. Biol. Theor. 6 4-15

Montévil M and Mossio M 2015 Biological organisation as closure of constraints. J. Theor. Biol. 372 179-191

Moore A 2012 Life defined. BioEssays 34 253-254

Mora T and Bialek W 2011 Are biological systems poised at criticality? J. Stat. Phys. 144 268-302

Moss L 2003 What genes can't do (Cambridge, MA: MIT Press)

Mossio M and Moreno A 2010 Organisational closure in biological organisms. Hist. Philos. Life Sci. 32 269-288

Muller GB 2003 Embryonic motility: environmental influences and evolutionary innovation. Evol. Dev. 5 56-60

Newman SA 2012 Physico-genetic determinants in the evolution of development. Science 338 217-219

Nicolis G and Prigogine I 1977 Self-organization in nonequilibrium systems (New York: Wiley)

Nykter M, Price ND, Aldana M, Ramsey SA, Kauffman SA, Hood LE, Yli-Harja O and Scmulevich I 2008 Gene expression dynamics in the macrophage exhibit criticality. Proc. Natl. Acad. Sci. USA 105 1897-1900

O’Malley MA and Dupre J 2005 Fundamental issues in systems biology. BioEssays 27 1270-1276

Rehen SK, McConnell MJ, Kaushal D, Kingsbury MA, Yang AH and Chun J 2001 Chromosomal variation in neurons of the 


\section{Giuseppe Longo et al.}

developing and adult mammalian nervous system. Proc. Natl. Acad. Sci. USA 98 13361-13366

Rot-Nikcevic I, Downing KJ, Hall BK and Kablar B 2007 Development of the mouse mandibles and clavicles in the absence of skeletal myogenesis. Histol. Histopathol. 22 51-60

Rubin H 1988 Can we speak of a mechanism for malignant transformation?'; in Theories of carcinogenesis: facts, fashion or fiction? (Washington, DC: Taylor and Francis) pp 31-43

Rubin H and Hatie C 1968 Increase in the size of chick embryo cells upon cultivation in serum-containing medium. Dev. Biol. 17 603-616

Saçlioglu C, Pekcan Ö and Nanjundiah V 2014 Group behaviour in physical, chemical and biological systems. J. Biosci. 39 177-189

Salam A 1990 Unification of fundamental forces (Cambridge: Cambridge University Press)

Shyer AE, Tallinen T, Nerukar NL, Wei Z, Gil ES, Kaplan DL, Tabin CJ and Mahadevan L 2013 Villification: how the gut gets its villi. Science 342 212-218

Sonnenschein C and Soto AM 1999 The society of cells: cancer and control of cell proliferation (New York: Springer Verlag)

Sonnenschein C, Soto AM and Michaelson CL 1996 Human serum albumin shares the properties of estrocolyone-I, the inhibitor of the proliferation of estrogen-target cells. J. Steroid Biochem. Mol. Biol. 59 147-154

Soto AM and Sonnenschein C 1985 The role of estrogens on the proliferation of human breast tumor cells (MCF-7). J. Steroid Biochem. 23 87-94

Soto AM and Sonnenschein C 2010 Environmental causes of cancer: endocrine disruptors as carcinogens. Nat. Rev. Endocrinol. 6 363-370

Soto AM and Sonnenschein C 2011 The tissue organization field theory of cancer: a testable replacement for the somatic mutation theory. BioEssays 33 332-340
Soto AM and Sonnenschein C 2012 Is systems biology a promising approach to resolve controversies in cancer research? Cancer Cell Int. 1212

Stebbings H 2001 Cell motility. Encyclopedia of life sciences (Nature Publishing Group) (www.els.net)

Steele M and Penny D 2010 Origins of life: common ancestry put to the test. Nature 465 168-169

Taniguchi Y, Choi PJ, Li GW, Chen H, Babu M, Hearn J, Emili A and Xie XS 2010 Quantifying E. coli proteome and transcriptome with single-molecule sensitivity in single cells. Science 329 533-538

Turing AM 1950 Computing machinery and intelligence. Mind 59 433-460

Turing AM 1952 The chemical basis of morphogenesis. Philos. Trans. R. Soc. Lond. [Biol]. 237 37-72

Tyagi S 2010 Genomics. E. coli, what a noisy bug. Science 329 518-519

Ueda H, Yokota E, Kutsuna N, Shimada T, Tamura K, Shimmen T, Hasezawa S, Dolja W, et al. 2010 Myosin-dependent endoplasmic reticulum motility and F-actin organization in plant cells. Proc. Natl. Acad. Sci. USA $1076894-6899$

van Doorn WG and van Meeteren U 2003 Flower opening and closure: a review. J. Exp. Bot. 54 1801-1812

van Fraassen BC 1989 Laws and symmetry (Oxford: Oxford University Press)

Varela FG, Maturana HR and Uribe R 1974 Autopoiesis: the organization of living systems, its characterization and a model. Biosystems 5 187-196

Werner G 2007 Metastability, criticality and phase transitions in brain and its models. Biosystems 90 496-508

Ying QL, Wray J, Nichols J, Batlle-Morera L, Doble B, Woodgett J, Cohen P and Smith A 2008 The ground state of embryonic stem cell self-renewal. Nature 453 519-523

MS accepted 20 April 2015; accepted 17 August 2015

Corresponding editor: SCOTT F GILBERT 